certificates were ascertained through December 31, 2007. Cancer diagnoses from 1988 through 2010 were identified by the Minnesota Cancer Surveillance System (MCSS). Standardised mortality ratios (SMRs) and proportional cancer incidence ratios (PCIR) were calculated using Minnesota as the reference population.

Results We identified 9,012 deaths, of which 2,693 were cancers, including 943 lung cancers, and 30 mesotheliomas. Mortality from all causes was near unity $(\mathrm{SMR}=1.02$, 95\% Confidence Interval (CI): 1.00-1.04). Mortality from lung cancer and mesothelioma were higher than expected; SMR $=1.16$ (95\% CI: 1.09-1.24) and 2.79 (95\% CI: 1.88-3.98) respectively. SMRs were elevated for all heart diseases $(\mathrm{SMR}=1.10,95 \%$ CI: 1.06-1.14), hypertension with heart disease $(\mathrm{SMR}=1.79$, 95\% CI: 1.37-2.30) and ischaemic heart disease ( $\mathrm{SMR}=1.11$, 95\% CI: 1.07-1.16). A total of 6,189 incident cancers were identified by MCSS including 1016 lung cancers, and 51 mesotheliomas. PCIRs for mesothelioma and lung cancer were 3.02 (95\% CI: 2.24, 3.98) and 1.22 (95\% CI: 1.15-1.30) respectively. Other proportionally elevated cancers include oral, esophageal, stomach, laryngeal, and bladder cancers.

Conclusions This analysis indicates taconite workers have an increased risk for certain cancers and cardiovascular disease. Exposures from taconite operations include crystalline silica, respirable dust and elongated mineral particles, including nonasbestiform amphiboles and cleavage fragments. Exposure to commercial asbestos is also possible. The extent to which such exposures contribute to disease burden is being investigated. Lifestyle factors, such as smoking, could also contribute to disease burden.

\section{A CASE-CONTROL STUDY OF MESOTHELIOMA IN MINNESOTA IRON ORE MINERS}

C L Lambert, Alexander, Mandel, Perlman, Ramachandran, MacLehose, Nelson. University of Minnesota, Minneapolis, United States of America

\subsection{6/oemed-2013-101717.343}

Objectives An excess of mesothelioma has been found in iron ore miners in northeastern Minnesota. Miners of taconite, the current form of ore mined, face a number of potentially hazardous exposures. The intensive processing of taconite ore combined with the unique and variable geology of the Mesabi Iron Range create the potential for exposure to natural amphibole fibres, cleavage fragments, and respirable dust. Exposure differed in natural ore (hematite) mining where minimal processing was required. We performed a case-control study to evaluate the risk of mesothelioma associated with taconite mining employment.

Methods Mesothelioma cases were identified through the Minnesota Cancer Surveillance System (MCSS) and death certificates from a cohort of 68,737 miners employed between the 1930s and 1982. Four controls of similar age were selected from the mining cohort for each case using an incidence density sampling protocol. Odds ratios and 95\% confidence intervals were estimated with conditional logistic regression for years employed in all iron ore mining, taconite mining, and the earlier natural ore mining.

Results Eighty cases of mesothelioma were identified in this cohort. There is a slight elevation of mesothelioma risk for each additional year of work in iron ore mining $(\mathrm{OR}=1.02,95 \% \mathrm{CI}$ $1.00-1.04)$ and taconite mining $(\mathrm{OR}=1.03$, 95\%CI $1.00-$ 1.07). No association was observed for years of employment in natural ore mining.
Conclusion These initial findings indicate a potential relationship between mesothelioma and employment in taconite mining and processing. The extent to which these cases are caused by exposure to taconite dust components, including cleavage fragments and non-fibrous amphiboles found in some zones of the Mesabi Range, or commercial asbestos will be explored in future analyses.

\section{Session: 23. Biological agents}

\section{WHAT IS THE OCCUPATIONAL RISK FOR TRANSMISSION OF HELICOBACTER PYLORI TO HEALTHCARE WORKERS WORKING IN INSTITUTIONS FOR THE ELDERLY?}

${ }^{1}$ A A De Schryver, ${ }^{2}$ Van Hooste, ${ }^{2}$ Charlier, ${ }^{2}$ Colemonts, ${ }^{3}$ Hambach, ${ }^{2}$ Schouteden, ${ }^{3}$ Van Sprundel. 'IDEWE/Universiteit Antwerpen, Leuven, Belgium; ${ }^{2}$ IDEWE, Leuven, Belgium; ${ }^{3}$ University of Antwerpen, Antwerp, Belgium

\subsection{6/oemed-2013-101717.344}

Background and Objectives Helicobacter pylori was discovered in 1984, but its transmission is not yet clear. Direct person-toperson transmission is most likely and could be relevant to occupational transmission particularly in healthcare workers (HCWs) in institutions for elderly people (IEP). Prevalence of H.pylori increases with age, and studies have shown a prevalence up to $85 \%$ in people living in IEP.

Methods We compared the occupational risk for $H$. pylori in HCWs working in IEP $(\mathrm{n}=198)$ to non-exposed controls $(\mathrm{n}=$ 250) in a cross-sectional study using serology. We calculated crude and age-adjusted prevalence; in bivariate analysis we compared both groups for a number of established general life-style and occupational risk factors. We applied logistic regression, Poisson regression with robust variance estimator and binomial regression, using established risk factors as covariates.

Results The crude prevalence of seropositivity was 14.6\% (95\% CI 9.7-19.6) in HCWs en $13.6 \%$ (95\%CI 9.4-17.8) in controls. Age-standardised prevalence was $14.2 \%$ versus $14.9 \%$ (difference not significant) respectively. Univariate analysis of seropositivity showed no significant associations with frequency of faecal contact, washing and feeding of elderly persons, contact with vomit, handling nasogastric tubes or washing linen. Using logistic regression, OR was 0.9 (95\% CI 0.5 - 1.6) in HCWs compared to non-exposed controls after adjusting for other risk factors; only age (OR $3.2-95 \%$ CI 1.6-6.3) and travel to developing countries (OR 2.0 $\quad$-CI $1.1-3.9$ ) predicted seropositivity to H. pylori. In Poisson and log binomial regression, prevalence ratio (PR) was 0.9 (95\%CI $0.6-1.5$ ) and 0.9 (95\%CI 0.6-1.4); only age and travel contributed significantly to H.pylori seropositivity in both models.

Conclusions In our study, using 3 multivariate methods (logistic regression, Poisson regression and binomial regression), the prevalence of H.pylori seropositivity was not significantly higher in HCWs compared to non-exposed controls. No significant associations between H.pylori seropositive status and occupational exposures were shown.

\section{PATTERNS OF S.AUREUS AND MRSA COLONIZATION AND INFECTION IN US SWINE VETERINARIANS}

R Davies, Sreevatsan,Sun, Yang. University of Minnesota, St. Paul, United States of America

10.1136/oemed-2013-101717.345 